\title{
DIFFÉRENCES ENTRE FAMILLES POUR LE DIMORPHISME SEXUEL DU POIDS À UN ÂGE DONNÉ CHEZ LA POULE ()
}

\author{
H. AYOUB et P. MÉRAT \\ Laboratoire de Génétique factorielle, \\ Centre national de Recherches zootechniques, I. N. R. A., \\ 78 - Jouy-en-Josas
}

\section{RÉSUMÉ}

Des différences d'amplitude limitée mais significatives ont été trouvées entre familles de même père (demi-frères, demi-sœurs) et entre familles de frères-sœurs intra-pères, pour le dimorphisme sexuel du poids à 4 semaines, 8 semaines et Io mois d'âge.

Ces différences sont mises en évidence dans des souches d'origines diverses, en testant l'existence d'interactions entre familles et sexe. L'interprétation génétique des résultats (gènes à manifestation différente suivant le sexe ou gènes liés au sexe) est discutée. Un effet d'échelle dû à la différence de variance entre sexes ne suffit apparemment pas à les expliquer entièrement.

\section{INTRODUCTION}

Le but du présent travail est de rechercher l'existence de variations génétiques dans le dimorphisme sexuel du poids chez la Poule. L'intérêt de la réduction de ce dimorphisme par sélection est important dans le domaine de la production des poulets de chair, où le fait que les femelles soient plus petites que les mâles vers l'âge de 8 semaines constitue un handicap commercial.

Les deux sexes reçoivent le même échantillon de gènes autosomaux de la génération parentale en l'absence de sélection. L'existence d'un dimorphisme sexuel peut avoir pour origine une sélection en faveur de gènes responsables d'une différence entre sexes, comme en témoignent quelques expériences de sélection. Une autre hypothèse veut y voir la manifestation de gènes liés au sexe, dont la répartition chez les mâles et les femelles est nécessairement différente. Enfin, un effet différentiel d'hétérosis peut intervenir, dans le cas du croisement.

Des données expérimentales sur l'un ou l'autre de ces points ont été recueillies par divers auteurs chez des oiseaux.

(1) Ce travail représente une partie d'une thèse de Docteur-Ingénieur soutenue par H. AyouB à Paris en r970. 


\section{A. - Expériences de sélection pour le dimorphisme sexuel ou identification de gènes modifiant ce dimorphisme}

Yoshida et Colnins (I967) ont étudié chez la caille japonaise le poids du corps à 8 semaines. L'expérience comportait trois lignées. L'une servait de contrôle. Dans la deuxième, les mâles seuls étaient sélectionnés pour des poids corporels élevés. Dans la dernière, c'étaient les femelles qui étaient soumises à cette sélection.

L'héritabilité du caractère a été calculée séparément pour chaque sexe et trouvée égale à 0,59 pour les mâles et à 0,47 pour les femelles. Les réponses à la sélection permettent d'estimer les héritabilités réalisées suivantes : 0,38 pour les mâles et 0,36 pour les femelles. Quant à l'héritabilité de la différence entre sexes, elle avait pour valeur 0,09 . Enfin, la corrélation génétique entre les poids à quatre semaines chez les femelles et chez les mâles était de 0,90 . En fin de compte, les différences entre sexes n'ont pas été modifiées par la sélection.

Horton et MCBride (I964) ont étudié chez la Poule le dimorphisme sexuel pour le poids à 4 et 8 semaines et le gain de poids entre ces âges. Deux souches sont gardées comme témoins. Neuf autres sont sélectionnées pour le poids à 8 semaines, deux d'entre elles pour accroître le dimorphisme sexuel, une autre pour le diminuer, les individus choisis étant accouplés au hasard. Les résultats mettent en évidence une interaction souche-sexe significative $(P<0,0 I)$.

Les corrélations génétiques entre mâles et femelles calculées entre pères intrasouches sont de l'ordre de 0,5 , significativement différentes de $\mathrm{r}$. La réponse à la sélection est plus élevée chez les mâles que chez les femelles, de sorte que le dimorphisme sexuel varie. Les auteurs estiment que la différence observée entre sexes était due à l'influence de gènes autosomaux.

Les expériences de sélection ou les analyses biométriques suggèrent donc dans certains cas la présence de gènes modifiant le rapport du poids des sexes, mais sans les identifier. On connaît au moins un tel gène chez la Poule. Il s'agit de dw (HUTT, I959) qui réduit la taille par rapport au phénotype "normal ", mais de façon différente dans les deux sexes.

\section{B. - Effet d'hétérosis}

Certaines recherches ont comparé les différences entre sexes dans les races pures et dans leurs croisements.

WESSELS et WILBRAHAM (I962) ont remarqué que, chez les canards, les différences entre sexes ont tendance à être plus importantes dans les croisements que dans les souches pures. Ils ont utilisé une souche de White Pekin comme souche mâle et Khaki Campbell comme souche femelle.

STONAKER (I963) réalise une expérience de croisements chez des bovins de race Hereford. Les mâles et les femelles sont issus de deux lignées consanguines. Les données portent sur I 229 veaux. Inbred et croisés étaient des demi-frères paternels. Les poids étaient mesurés à l'âge de 5 et 7 mois. L'étude des moyennes montre qu'il existe un effet d'hétérosis plus grand chez les femelles à 1'âge de 200 jours. Les différences entre sexes étaient de $\mathrm{I} 2 \mathrm{p}$. Ioo chez les inbred et $5 \mathrm{p}$. IOo chez les hybrides. STONAKER explique ce phénomène par le fait que les femelles (homogamétiques) ont plus de loci en disjonction que les mâles (hétérogamétiques).

Le même auteur a été amené par ces résultats à examiner le problème dans les 
espèces où c'est le mâle qui est homogamétique (dinde, poule). En ce qui concerne la Dinde, il étudie des résultats obtenus par CLARK (I960) et Moreng et Thornton (I958) : Ce sont ici les mâles qui présentent le plus grand effet d'hétérosis ; la valeur relative des croisés par rapport aux inbred est de I,09 chez les mâles et I, or chez les femelles. L'interaction sexe $\times$ système d'élevage est plus importante que chez les bovins. Quant à la Poule, il utilise les résultats de GLAZENER et al. (I95I). Dans leur expérience, 8 lignées consanguines issues de 4 souches (White Leghorn, Rhode Island Red, Barred Plymouth Rock et New Hampshire) sont croisées avec des femelles non consanguines comprenant une souche White Plymouth Rock et les 4 souches mentionnées. La supériorité relative des croisés par rapport aux lignées consanguines était en moyenne de $\mathrm{I}, \mathrm{I}_{4}$ pour les mâles et $\mathrm{I}, 08$ pour les femelles.

Ces résultats montrent que 1'interaction souche-sexe est pour une large part expliquée par 1'action des gènes liés au sexe. Le coefficient de régression du poids sur le coefficient de consanguinité est plus grand chez le sexe homogamétique dans les espèces étudiées. STONAKER a proposé d'appeler ce phénomène " hétérosis homogamétique ". Cependant, on peut noter que tous les résultats ne semblent pas s'accorder avec ce schéma (par exemple WHITE et al., I968).

\section{C. - Mise en évidence de l'effet de gènes liés au sexe}

Certaines recherches ont mis en évidence l'influence des gènes liés au sexe sur le dimorphisme sexuel.

SHAKLEE et al. (I952) ont fait une expérience sur dindes en croisant 24 mâles et I42 femelles Beltsville White et ont obtenu 852 descendants.

Ils ont étudié le poids du corps à l'âge de 24 semaines. Il s'agissait d'une sélection familiale pour améliorer la production de viande et des caractères de reproduction. Le poids de chaque femelle était retranché du poids moyen de ses frères et vice-versa. L'analyse de la variance montre que l'effet de la mère est significatif. Les familles de frères-sœurs ne sont pas équivalentes quant à la différence de poids entre sexes.

Cette différence est donc influencée par l'hérédité, et peut-être en particulier par des gènes liés au sexe. Le coefficient de variation, par ailleurs, est plus élevé chez les femelles que chez les mâles.

D'autre part, les données de ces mêmes auteurs démontrent que dans les croisements la vitesse de croissance des mâles est relativement plus importante (STONAKER, I963).

BEILHARZ (I960) donne une interprétation de l'effet possible de gènes liés au sexe dans les résultats de SHAKLEE : le père apporte un chromosome $\mathrm{X}$ aux femelles ainsi qu'aux mâles et son influence est la même dans les deux sexes. En revanche, les mères donnent le chromosome $\mathrm{X}$ uniquement à leurs fils. Ceci peut changer l'importance relative des pères par rapport aux mères, en fonction de l'importance génétique du chromosome $\mathrm{X}$.

BEILHARZ étudie ce problème chez le Lapin (à l'âge de I6 semaines dans un groupe de 429 lapins) et chez le Poulet, sur 3 populations (Australorp, White Leghorn et croisements réciproques). Les résultats suggèrent un rôle joué par des gènes liés au sexe dans tous les cas; en particulier, en croisant les pères légers avec des mères 
lourdes, la différence entre les deux sexes est plus élevée que dans le cas du croisement réciproque.

Des gènes liés au sexe ayant un effet sur la taille, et en conséquence sur son dimorphisme sexuel par leur répartition dans les deux sexes, ont été identifiés directement. Nous avons cité $d w$; un autre facteur issu des souches naines de type Bantam est connu également (MAw, I935; GoDfrey, I953; JAAP, I969).

Dans le présent travail, nous mettrons en évidence des variations génétiques du dimorphisme sexuel pour le poids à divers âges, entre familles à l'intérieur de plusieurs souches.

\section{MATÉRIEL}

Parmi les souches étudiées, certaines étaient élevées à Jouy-en-Josas (population a Jouy " issue du croisement de plusieurs souches : MERAT, 1962; une souche de Faverolles; souche " $L 22$ " synthétique issue d'un croisement initial).

Au domaine du Magneraud, les résultats obtenus portent sur 4 souches, en Ig66 ou I968 (cf Tableau I).

L'élevage était fait au sol pour les jeunes et les adultes. Les deux sexes étaient élevés ensemble en poussinière. Après 8 semaines d'âge, toutes les poulettes étaient gardées jusqu'à l'âge adulte (ro mois environ), du moins dans les familles les plus nombreuses. Pour les coqs, une fraction était éliminée à 8 semaines, en partie d'après le poids à cet âge, à l'exception de certains cheptels du Magneraud qui seront mentionnés séparément. Dans chaque sexe, à l'intérieur des diverses souches analysées, toutes les familles étaient distribuées ensemble dans les mêmes locaux.

\section{MÉTHODES}

Pour mettre en évidence des différences entre familles pour le dimorphisme sexuel relativement à un caractère donné, nous avons utilisé le test d'interaction entre familles et sexe pour ce caractère.

L'étude des différences entre familles de même père (demi-frères et demi-sœurs) est faite, suivant les souches et les générations, pour un ou plusieurs des caractères suivants : Poids à l'âge de 4 semaines, de 8 semaines, poids " adulte " à Io mois. Le choix de l'analyse sur l'un ou l'autre caractère dans un cheptel était fonction, soit des données disponibles, soit de l'importance des effectifs pour chaque mesure. Le tableau $\mathrm{I}$ rassemble les souches, années et effectifs sur lesquels porte notre analyse, par caractère.

A partir de ces données, une analyse de variance est faite intra-années et troupeaux, avec les deux facteurs contrôlés " sexe " et "famille de même père ".

L'interaction famille $\times$ sexe a été testée par la méthode approchée tenant compte de la présence d'effectifs différents dans les groupes élémentaires (SNEDECOR et COCHRAN, I967).

Une difficulté accessoire pour la validité de l'analyse de variance provenait de la différence de variance résiduelle existant entre mâles et femelles ; toutefois, il a été possible de ne pas en tenir compte, cette différence de variance n'étant pas très considérable.

Notre analyse du poids adulte, dans la population Jouy, ne porte pas directement sur lui, mais sur sa différence, pour chaque individu, avec le poids à 8 semaines. En effet, un tri a été fait parmi les mâles à 8 semaines, la "sévérité " relative de ce tri étant variable. Il en résulte, du fait que le poids à 8 semaines représente une partie du poids adulte et présente une corrélation appréciable avec lui, un "biais" variable dans les différences entre sexes pour ce poids adulte. Par contre, la corrélation entre poids à 8 semaines et croissance de 8 semaines à l'âge adulte varie suivant l'année de 0,035 à 0,270 pour les femelles et de 0,055 à 0,396 chez les mâles, de sorte que le "biais " en question, pour cette croissance après 8 semaines, ne risque pas d'être très important ni surtout de différer beaucoup suivant les familles. Nous nous sommes d'ailleurs limités, dans la souche Jouy, à quatre années où un nombre suffisant de mâles étaient gardés jusqu'à ro mois.

Une analyse directe sur ce caractère a pu être faite sur les cheptels élevés au domaine du Magneraud, où la plupart des mâles avaient été gardés après l'âge de 8 semaines. Deux souches 
" femelle chair ", $J 11$ et $J 55$, l'une naine, possédant le gène dw (Hutr, I959), l'autre normale, comparées la même année (1968); et deux souches " ponte ", $M 11$ en r968 et $M$ r7 en 1966.

D'autre part, une étude par familles de même mère a été faite dans les quatre souches du Magneraud indiquées dans le tableau précédent, ainsi que dans une année (1967) de la population de Jouy-en-Josas, ces données comportant des familles de frères-sœurs suffisamment nombreuses. De cette étude, nous avions supprimé les familles ayant moins de 5 descendants de même sexe. L'analyse de variance, faite ici sur une base intra-père, comportait les facteurs contrôlés "sexe " et " famille de même mère " (frères-sœurs).

\section{TABLEAU I}

Populations, années et nombre d'animaux analysés dans l'étude par familles de même père

\begin{tabular}{|c|c|c|c|c|c|c|c|}
\hline \multirow{2}{*}{ Souches } & \multirow{2}{*}{ Années } & \multicolumn{2}{|c|}{4 semaines } & \multicolumn{2}{|c|}{8 semaines } & \multicolumn{2}{|c|}{10 mois (adultes) } \\
\hline & & $\widehat{\partial \sigma}$ & 우우 & केర & 우우 & वैర & 우우 \\
\hline \multirow[t]{2}{*}{ Jouy-en-Josas } & $\begin{array}{l}1959 \\
1960 \\
1961 \\
1962 \\
1963 \\
1964 \\
1965 \\
1966 \\
1967\end{array}$ & $\begin{array}{rr}1 & 099 \\
1 & 122 \\
1 & 524 \\
1 & 069 \\
1 & 411 \\
& 746 \\
& 723 \\
& 676 \\
& 975\end{array}$ & $\begin{array}{rl}1 & 147 \\
1 & 144 \\
1 & 538 \\
1 & 068 \\
1 & 415 \\
712 \\
704 \\
705 \\
\\
\\
944\end{array}$ & $\begin{array}{rl}1 & 060 \\
1 & 193 \\
1 & 500 \\
1 & 018 \\
1 & 311 \\
728 \\
727 \\
675 \\
\\
& 963\end{array}$ & $\begin{array}{rr}1 & 033 \\
1 & 137 \\
1 & 519 \\
1 & 039 \\
1 & 344 \\
& 707 \\
& 777 \\
& 706 \\
& 934\end{array}$ & $\begin{array}{l}48 \\
41 \\
27 \\
55\end{array}$ & $\begin{array}{r}254 \\
134 \\
67 \\
231\end{array}$ \\
\hline & Total & 9345 & 9447 & 9175 & 9196 & 171 & 686 \\
\hline \multirow[t]{2}{*}{ Faverolles } & $\begin{array}{l}1960 \\
1961 \\
1962 \\
1963\end{array}$ & $\begin{array}{l}795 \\
369\end{array}$ & $\begin{array}{l}739 \\
376\end{array}$ & $\begin{array}{l}595 \\
316 \\
768 \\
368\end{array}$ & $\begin{array}{l}581 \\
351 \\
711 \\
376\end{array}$ & & \\
\hline & Total & 1164 & 1115 & 2047 & 2019 & - & - \\
\hline \multirow[t]{2}{*}{ " $L 22 "$} & $\begin{array}{l}1963 \\
1964\end{array}$ & $\overline{649}$ & $\overline{703}$ & $\begin{array}{l}578 \\
617\end{array}$ & $\begin{array}{l}549 \\
658\end{array}$ & & \\
\hline & Total & 649 & 703 & 1195 & 1207 & & \\
\hline \begin{tabular}{l}
\multicolumn{2}{c}{ Magneraud } \\
$J \quad 55 \ldots \ldots \ldots \ldots \ldots$ \\
$J 11 \ldots \ldots \ldots \ldots$ \\
$M 11 \ldots \ldots \ldots$ \\
$M \quad 77 \ldots \ldots \ldots \ldots$
\end{tabular} & $\begin{array}{l}1968 \\
1968 \\
1966 \\
1968 \\
\text { Total }\end{array}$ & & & & & $\begin{array}{r}172 \\
251 \\
1806 \\
277 \\
2506\end{array}$ & $\begin{array}{r}764 \\
870 \\
1970 \\
607 \\
4211\end{array}$ \\
\hline
\end{tabular}

Eisen et Legates (I966) ont fait remarquer que le test d'interaction souches $\times$ sexe est sujet à une difficulté d'interprétation, la composante "interaction " de la variance contenant un terme fonction de la différence de la variance génétique dans les deux sexes. Nous avons néanmoins utilisé ce test, mais indiquons plus loin, par une estimation approximative du terme correctif dans les divers cas étudiés, que cet effet " d'échelle " associé à la différence de variance chez les mâles et les femelles peut difficilement rendre compte à lui seul des interactions significatives que nous observons.

Enfin, l'héritabilité du dimorphisme sexuel du poids à 8 semaines a été estimée dans deux années de la population Jouy, I966 et 1967. La variable étudiée est la différence, pour chaque mère, entre le poids moyen des descendants mâles et celui des femelles. Des nombres égaux ont 
été gardés pour les deux sexes dans chaque famille, correspondant en I966 à 7 pères, I6 mères et 72 descendants de chaque sexe, en 1967 à I 3 pères, 38 mères et $I 60$ descendants de chaque sexe.

La méthode est celle utilisée par KinNey et Shoffner (I965) et Buvanendran(I969).

Le modèle mathématique est le suivant :

$$
\bar{p}_{i j}=\mu+s_{i}+d_{i j}+e_{i j}
$$

où $\bar{p}_{i j}$ est la valeur observée pour la variable attachée à la ième mère et au ième père

$\mu$ est la moyenne de la population

$s_{i}$ est un effet commun aux descendants du $i$ ième père

$d_{i j}$ est un effet commun auxdescendants de la $j$ ième mère accouplée au i ième père

$e_{i j}$ un écart aléatoire provenant de l'échantillonnage des descendants de la jième mère.

Le tableau d'analyse de la variance est le suivant (tabl. 2) :

\section{TABLEAU 2}

Tableau d'analyse de la variance pour l'estimation de l'héritabilité de la différence de poids entre sexes

\begin{tabular}{l|c|c}
\hline Source de variation & $\begin{array}{c}\text { Degrés } \\
\text { de liberté }\end{array}$ & $\begin{array}{c}\text { Espérance mathématique } \\
\text { de la variance }\end{array}$ \\
\hline Péres.......... & $s-\mathrm{r}$ & $\mathrm{K}_{2} \sigma_{e}^{2}+\sigma_{d}^{2}+\mathrm{K}_{3} \sigma_{s}^{2}$ \\
Mères intra pères ... & $d-s$ & $\mathrm{~K}_{1} \sigma_{e}^{2}+\sigma_{d}^{2}$ \\
\hline
\end{tabular}

Dans ce tableau, $\sigma_{e}^{2}, \sigma_{d}^{2}, \sigma_{\delta}^{2}$ représentent respectivement les composantes " résiduelles ", " mère " et " père " de la variance, $d$ et $s$ sont respectivement les nombres de mères et de pères, $K_{1}, K_{2}$ et $K_{3}$ sont des coefficients calculés d'après les formules indiquées par KINNEY et SHOFFNER (1965).

Pour $\sigma_{\ell}^{2}$, nous utilisons l'estimation proposée par Buvanendran (I969) :

$$
\sigma_{e(1-2)}^{2}=\sigma_{(1)}^{2}+\sigma_{(2)}^{2}-2 \operatorname{cov}(\mathrm{r} 2)
$$

(I) et (2) correspondant respectivement aux mâles et aux femelles, en tenant compte de ce que l'espérance mathématique de cov (12) est nulle, $\sigma_{(\mathbf{1})}^{2}$ et $\sigma_{(2)}^{2}$ étant estimés à partir de l'analyse de variance faite séparément dans chaque sexe $\left(^{1}\right)$.

L'héritabilité du dimorphisme sexuel basée sur la composante " père " est alors donnée par

$$
h^{2}=\frac{4 \sigma_{s}^{2}}{\sigma_{s}^{2}+\sigma_{d}^{2}+\sigma_{e}^{2}}
$$

\section{RÉSULTATS}

\section{I. - Différences entre familles de pères}

\section{a) Poids à 4 semaines.}

Pour la population Jouy, la valeur moyenne de ce critère varie entre 204 et

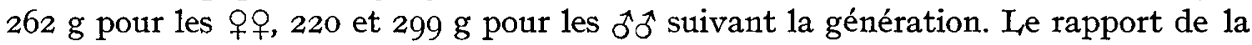

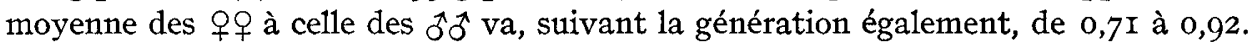
La variance est plus élevée chez les $\widehat{0} \sigma^{\wedge}$.

(1) Il ne nous a pas paru utile d'indiquer les estimations de $\sigma_{e}^{2}$ dans la présentation des résultats (tabl. ro). 
Pour les souches Faverolles et $L 22$, ces données sont du même ordre de grandeur.

Le tableau 3 contient, par année et pour chaque souche analysée, les degrés de liberté de la variance interaction père $x$ sexe et de la variance résiduelle ainsi que le rapport de la première à la seconde. Pour l'alléger, nous avons omis les variances " entre sexes » et " entre pères ", toutes deux hautement significatives dans l'ensemble comme on pouvait s'y attendre.

\section{TABLEAU 3}

Poids à 4 semaines, population Jouy, souches Faverolles et $\mathrm{L} 22$ Variance interaction pères $\times$ sexe comparée à la variance résiduelle par année

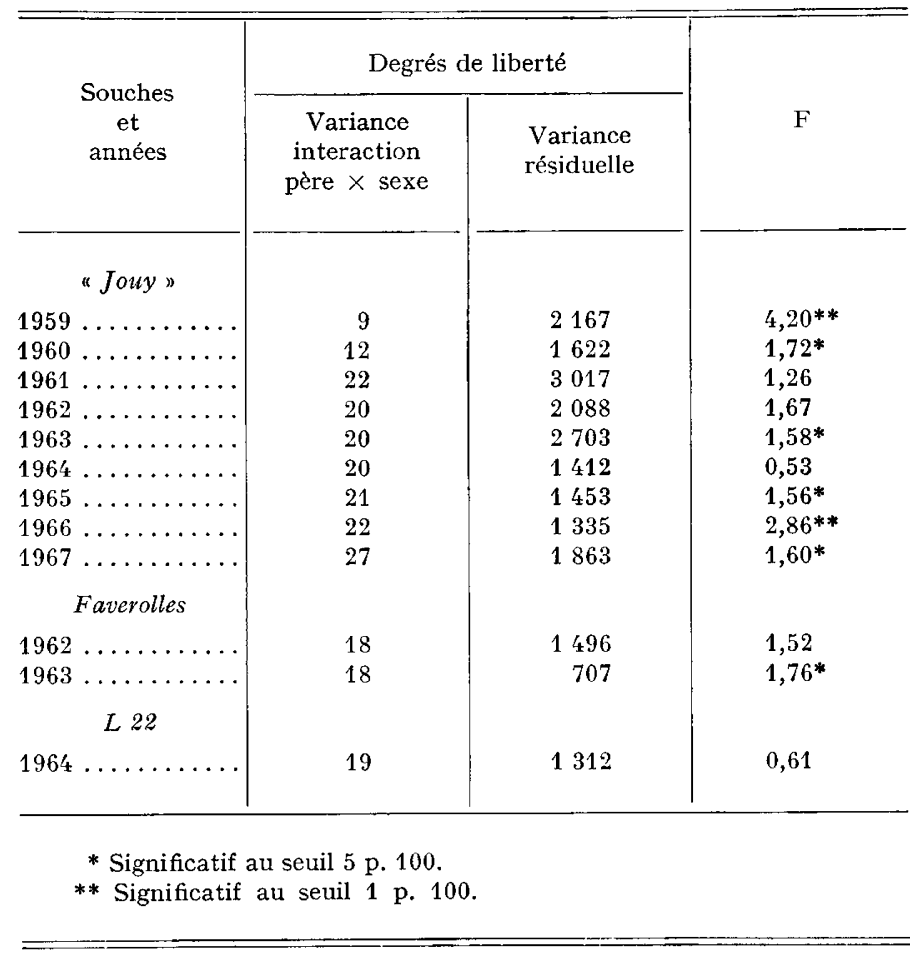

Dans la souche Jouy, l'interaction père $\times$ sexe est, 6 fois sur 9 , significative au moins au seuil $5 \mathrm{p}$. Ioo, et dans 8 cas le rapport $\mathrm{F}$ correspondant est supérieur à 1'unité. En groupant la variance interaction sur l'ensemble des années et la comparant à la variance résiduelle sur une base intra-année, on trouve un rapport $F$ égal à $x, 73$ pour I72 et I7 660 degrés de liberté, significatif au seuil I p. Ioo.

Pour la souche Faverolles également, dans l'ensemble, la variance interaction père $\times$ sexe est supérieure à la variance résiduelle (sur les deux années regroupées, $\mathrm{F}=\mathrm{I}, 45$ pour 36 et 2203 d.1., $\mathrm{P}<0,05)$.

On peut donc conclure à l'existence de variations entre familles de pères quant au dimorphisme sexuel du poids à 1'âge de 4 semaines. 


\section{TABLEAU 4}

Poids à 8 semaines: Variance interaction pères $\times$ sexe comparée à la variance résiduelle, par année

\begin{tabular}{|c|c|c|c|}
\hline \multirow{2}{*}{$\begin{array}{l}\text { Souches } \\
\text { et } \\
\text { années }\end{array}$} & \multicolumn{2}{|c|}{ Degrés de liberté } & \multirow[b]{2}{*}{$\mathrm{F}$} \\
\hline & $\begin{array}{c}\text { Variance } \\
\text { interaction } \\
\text { père } \times \text { sexe }\end{array}$ & $\begin{array}{l}\text { Variance } \\
\text { résiduelle }\end{array}$ & \\
\hline \multicolumn{4}{|l|}{ Jouy } \\
\hline $1959 \ldots \ldots \ldots$ & 9 & 2074 & 1,01 \\
\hline $1960 \ldots \ldots \ldots$ & 12 & 2214 & $2,14 *$ \\
\hline $1961 \ldots \ldots \ldots \ldots$ & 22 & 2955 & $2,07 * *$ \\
\hline $1962 \ldots \ldots \ldots$ & 20 & 2001 & $1,82 *$ \\
\hline $1963 \ldots \ldots \ldots \ldots$ & 20 & 2613 & 0,09 \\
\hline $1964 \ldots \ldots \ldots \ldots$ & 20 & 1354 & $1,92 * *$ \\
\hline $1965 \ldots \ldots \ldots \ldots$ & 21 & 1460 & 1,10 \\
\hline $1966 \ldots \ldots \ldots$ & 22 & 1331 & $1,53^{*}$ \\
\hline $1967 \ldots \ldots \ldots$ & 27 & 1840 & 1,30 \\
\hline \multicolumn{4}{|l|}{ Faverolles } \\
\hline $1960 \ldots \ldots \ldots$ & 21 & 1132 & $2,17 * *$ \\
\hline $1961 \ldots \ldots \ldots$ & 10 & 645 & 0,97 \\
\hline $1962 \ldots \ldots \ldots$ & 18 & 1436 & $1,80^{*}$ \\
\hline $1963 \ldots \ldots \ldots$ & 18 & 706 & 0,71 \\
\hline$L 22$ & & & \\
\hline $1963 \ldots \ldots \ldots$ & 17 & 1091 & 1,15 \\
\hline $1964 \ldots \ldots \ldots$ & 19 & 1268 & 0,76 \\
\hline
\end{tabular}

* Significatif au seuil 5 p. 100.

** Significatif au seuil 1 p. 100.

\section{TABLEAU 5}

Croissance de 8 semaines à l'âge adulte, population Jouy Test de l'interaction pères $\times$ sexe

\begin{tabular}{l|c|c|c}
\hline \multirow{2}{*}{ Année } & \multicolumn{2}{|c|}{ Degrés de liberté } & \\
\cline { 2 - 4 } & $\begin{array}{c}\text { Variance } \\
\text { interaction }\end{array}$ & $\begin{array}{c}\text { Variance } \\
\text { résiduelle }\end{array}$ & $\mathrm{F}$ \\
\hline & 13 & 274 & 1,61 \\
1964 & 9 & 155 & 1,72 \\
1965 & 6 & 80 & 1,23 \\
1966 & 13 & 258 & 1,39 \\
\hline \hline
\end{tabular}


b) Poids à 8 semaines.

Quant au poids à 8 semaines, sa moyenne par génération chez les femelles varie de 599 à $808 \mathrm{~g}$, chez les mâles de 687 à $\mathrm{I}$ or $8 \mathrm{~g}$, le rapport de la moyenne des premières à celle des seconds fluctuant entre 0,79 et 0,87 , donc moins élevé dans 1'ensemble et moins variable qu'à 4 semaines. Les valeurs sont du même ordre pour la Faverolles; la souche $L 22$ étant plus légère.

Le tableau 4 indique, par souche et par génération, le rapport de la variance interaction père $x$ sexe à la variance résiduelle.

Dans toutes les années de la souche Jouy sauf une, la variation interaction pères $\times$ sexe est supérieure à la variance résiduelle $(F>I)$. Une estimation globale intra-années de cette interaction donne un rapport $F=I, 43$ vis-à-vis de la variance résiduelle, avec les degrés de liberté I73 et I8 I77 (significatif au seuil I p. roo).

Dans la souche Faverolles, le test global d'interaction père $\times$ sexe intra-années correspond à 67 degrés de liberté pour la variance interaction et à 3 99I pour la variance résiduelle. Le rapport $\mathrm{F}$ est égal à $\mathrm{I}, 5 \mathrm{I}(\mathrm{P}<0,05)$. Par contre, pour la souche $L 22$, il n'y a pas d'interaction décelable.

Au total, dans ces données, le dimorphisme sexuel varie donc quelque peu suivant le père, à l'âge de 8 semaines comme à celui de 4 semaines.

\section{c) Poids adulte.}

I. Quant au dimorphisme sexuel de la croissance de 8 semaines à l'âge adulte, le rapport de la moyenne des 우오 à celle des $\widehat{o} \widehat{o}$ est compris entre 0,64 et 0,7 I suivant l'année.

Le tableau 5 présente pour ce caractère la comparaison entre variance interaction père $\times$ sexe et résiduelle (la variance due aux pères étant significative dans deux des quatre années, celle due au sexe l'étant toujours).

L'interaction n'est pas significative dans chaque cas pris isolément, mais le $F$ correspondant est toujours plus grand que I. Sur l'ensemble des 4 années, le rapport F de la variance interaction à la variance résiduelle, toutes deux estimées sur une base intra-année, avec respectivement $4 \mathrm{I}$ et 767 d.1., est égal à $\mathrm{I}, 47(\mathrm{P}<0,05)$.

2. L'analyse directe sur les poids adultes (test de l'interaction pères $\times$ sexe) est contenue dans le tableau 6 .

L'interaction père $\times$ sexe apparaît hautement significative dans les quatre souches analysées.

\section{II. - Différences entre familles de mères (frères-scurs)}

Parallèlement aux différences entre familles de même père, nous étudions dans ce qui suit les différences entre familles de frères-sœurs pour le dimorphisme sexuel du poids. A cet effet, une analyse de variance est faite, afin d'estimer l'interaction mères $X$ sexe intra-pères.

Cette analyse a été limitée aux troupeaux suivants présentant des effectifs suffisants par mères : population "Jouy " en I 967 pour le poids à l'âge de 8 semaines ; au Magneraud, souche $J 11$ naine en I968, souches $M$ 7/y et $M 11$ en Ig66 pour le poids adulte. 
TABLEAU 6

Poids adulte, données du Magneraud. Test de l'interaction pères $\times$ sexe

\begin{tabular}{|c|c|c|c|}
\hline \multirow[b]{2}{*}{ Souche et année } & \multicolumn{2}{|c|}{ Degrés de liberté } & \multirow[b]{2}{*}{$\mathrm{F}$} \\
\hline & $\begin{array}{c}\text { Variance } \\
\text { interaction }\end{array}$ & $\begin{array}{l}\text { Variance } \\
\text { résiduelle }\end{array}$ & \\
\hline$J 11$ (naine), $1968 \ldots$ & 15 & 1089 & $3,59^{* *}$ \\
\hline$J 55$ (normale), 1968 . & 16 & 902 & $4,50 * *$ \\
\hline$M 11,1966, \ldots \ldots \ldots$ & 18 & 3738 & $7,93^{* *}$ \\
\hline$M 77,1968 \ldots \ldots \ldots$ & 7 & 868 & $2,83^{* *}$ \\
\hline
\end{tabular}

** Significatif au seuil 1 p. 100.

TABLEAU 7

Analyse de la variance par sexe et familles de mères intra-pères Poids à 8 semaines, population Jouy 1967

\begin{tabular}{|c|c|c|}
\hline Source de variation & Degrés de liberté & $\mathrm{F}$ \\
\hline $\begin{array}{l}\text { Sexes } \ldots \ldots \ldots \\
\text { Mères intra-pères } \ldots \ldots \\
\text { Interaction mère } \times \text { sexe } \\
\text { intra-père } \ldots \ldots \ldots \ldots \\
\text { Résiduelle } \ldots \ldots \ldots \ldots\end{array}$ & $\begin{array}{r}1 \\
89 \\
\\
89 \\
1518\end{array}$ & $\begin{array}{r}418,72^{* *} \\
5,46^{* *} \\
1,35^{*}\end{array}$ \\
\hline
\end{tabular}

TABLEAU 8

Analyse de variance par sexe et familles de mères intra-pères: poids adulte, données du Magneraud

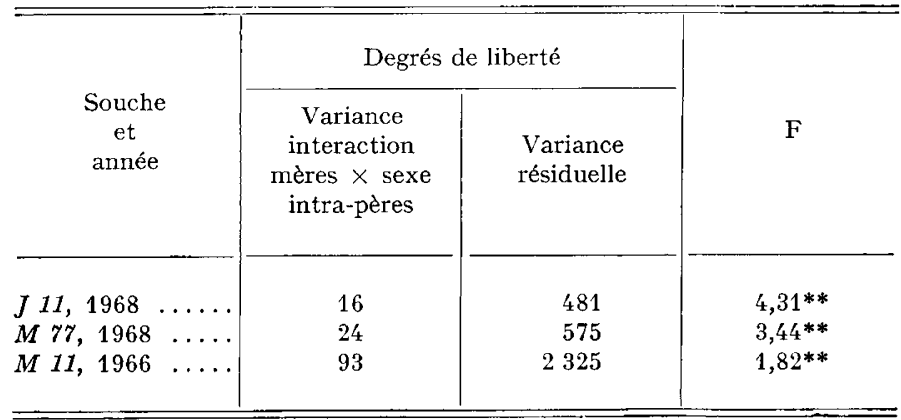


L'ensemble des résultats contenus dans les tableaux 7 et 8 permet de conclure que l'interaction mère $\times$ sexe intra-père est toujours significative pour le poids à 1'âge adulte, le degré de signification étant dans l'ensemble plus élevé que pour 1 'interaction pères $\times$ sexe.

Pour le seul cas étudié à l'âge de 8 semaines (population Jouy r967), l'interaction mères $\times$ sexe apparaît significative au seuil $5 \mathrm{p}$. Ioo, alors que, la même année, l'interaction pères $\times$ sexe n'était pas significative. Dans l'ensemble, ceci paraît confirmer que les variations entre mères pour le dimorphisme sexuel sont plus importantes que celles entre pères, en accord avec les observations d'autres auteurs (SHAKLEE et al., I952).

III. - Estimation de l'héritabilité du dimorphisme sexuel pour le poids à 8 semaines (Années I966 et I967, souche Jouy)

Le tableau 9 indique les héritabilités du poids, basées respectivement sur la composante " père " et " mère " de la variance, trouvées dans chaque sexe séparément, pour chaque année et pour le total.

TABLEAU 9

Héritabilité du poids à 8 semaines pour chaque sexe

\begin{tabular}{|c|c|c|c|c|c|c|}
\hline \multirow[b]{2}{*}{ Année } & \multicolumn{3}{|c|}{ 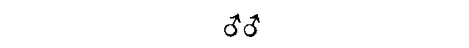 } & \multicolumn{3}{|c|}{ 우우 } \\
\hline & $\begin{array}{c}h_{s}^{2} \\
\text { (basée } \\
\text { sur la } \\
\text { composante } \\
\text { " père ") }\end{array}$ & $\begin{array}{c}h_{\mathcal{D}}^{2} \\
\text { (basée } \\
\text { sur la } \\
\text { composante } \\
\text { " mère ") }\end{array}$ & $\begin{array}{c}h^{2} \\
\text { moyenne } \\
\text { des deux }\end{array}$ & $h_{s}^{2}$ & $h_{\mathrm{D}}^{2}$ & $h^{2}$ \\
\hline $\begin{array}{l}1966 \\
1967\end{array}$ & $\begin{array}{l}0,03 \\
1,01\end{array}$ & $\begin{array}{l}1,45 \\
0,40\end{array}$ & $\begin{array}{l}0,76 \\
0,70\end{array}$ & $\begin{array}{l}1,00 \\
1,12\end{array}$ & $\begin{array}{l}0,42 \\
0,64\end{array}$ & $\begin{array}{l}0,71 \\
0,88\end{array}$ \\
\hline $\begin{array}{c}\text { Ensemble } \\
\text { (intra-années) }\end{array}$ & 0,60 & 0,74 & 0,67 & 0,97 & 0,60 & 0,78 \\
\hline
\end{tabular}

TABLEAU IO

Composantes de la variance et héritabilité du dimorphisme sexuel pour le poids à 8 semaines

\begin{tabular}{|c|c|c|c|c|}
\hline Année & $\begin{array}{c}\text { Source } \\
\text { de variation }\end{array}$ & $\begin{array}{c}\text { Degrés } \\
\text { de } \\
\text { liberté }\end{array}$ & $\begin{array}{c}\text { Composantes } \\
\text { de la } \\
\text { variance }\end{array}$ & $h_{s}^{2}$ \\
\hline 1966 & $\begin{array}{c}\text { pères - } \\
\text { mères intra-pères }\end{array}$ & $\begin{array}{r}6 \\
10\end{array}$ & $\begin{array}{c}(-13,01) \\
40,64\end{array}$ & $(-1,30)$ \\
\hline 1967 & $\begin{array}{c}\text { pères } \\
\text { mères intra-pères }\end{array}$ & $\begin{array}{l}12 \\
26\end{array}$ & $\begin{array}{r}3,57 \\
40,26\end{array}$ & 0,36 \\
\hline $\begin{array}{c}\text { Ensemble } \\
\text { (intra-années) }\end{array}$ & $\begin{array}{l}\text { pères } \\
\text { mères }\end{array}$ & $\begin{array}{l}18 \\
36\end{array}$ & $\begin{array}{c}(-1,91) \\
40,60\end{array}$ & $(-0,20)$ \\
\hline
\end{tabular}


On remarque, en particulier, que l'héritabilité basée sur la composante " père » de la variance $\left(h^{2}\right)$ est moins élevée en proportion chez les mâles que chez les femelles, ce qui s'accorde avec les résultats de MERRITT (I967).

Le tableau ro donne les valeurs estimées des composantes de la variance et de l'héritabilité basée sur la composante "père " en ce qui concerne le dimorphisme sexuel.

La valeur négative trouvée en I966, attribuable à l'échantillonnage et pouvant être considérée comme nulle, correspond à une très faible variation, entre familles, de la différence de poids entre sexes. Par contre, en I967, une valeur positive appréciable est notée.

\section{DISCUSSION ET CONCLUSIONS}

Nos résultats contribuent à mettre en évidence 1'existence de variations génétiques de la différence du poids moyen entre sexes :

- Interactions père $\times$ sexe hautement significatives dans l'ensemble, à 4 et 8 semaines, et pour la croissance après 8 semaines, sur les données de Jouy-en-Josas, à 1'âge adulte dans 3 souches du Magneraud, correspondant à des variations entre familles de même père pour le dimorphisme sexuel à ces différents stades.

- Interactions mère $\times$ sexe intra-père hautement significatives également, tant pour le poids à 8 semaines (Jouy) que pour le poids des animaux adultes.

Les variations observées pour le dimorphisme sexuel paraissent donc, au total, réelles, mais, il est vrai, d'amplitude assez limitée, comme cela se traduit d'une autre façon par les valeurs d'héritabilité trouvées par nous et d'autres chercheurs (Yoshidd et Colins, I967 ; Buvanendran, I969). Elles sont un peu plus marquées entre mères qu'entre pères, suggérant qu'une sélection par familles de frères-scurs pourrait influer sur ce caractère (cf. SHAKLEE et al. I952; BEILHARZ, I960).

Toutefois, EISEN et LEGATES (I966) ont indiqué la possibilité qu'une variation comme celle que nous observons ne soit qu'un artefact, causé par l'inégalité de variance entre les deux sexes.

D'après leur raisonnement théorique, on peut estimer une composante $\sigma_{\mathrm{G}}^{2} \mathrm{~s}^{\prime}$ " interaction sexe-groupe génétique " non biaisée de la façon suivante :

avec

$$
\sigma_{\mathrm{G}}^{2} \mathrm{~S}^{\prime}=\sigma_{\mathrm{G} S}^{2}-\mathrm{I} / 2\left(\sigma_{\mathrm{G}_{1}}-\sigma_{\mathrm{G}_{2}}\right)^{2}
$$

$$
\sigma_{\mathrm{GS}}^{2}=\frac{\sigma_{2}}{n}(\mathrm{~F}-\mathrm{I})
$$

$\sigma^{2}$ représentant la variance phénotypique résiduelle (intra-sexes et groupes)

$n$ représentant l'effectif de chaque groupe élémentaire

F le rapport de la variance "interaction sexe-groupe " à la variance résiduelle $\sigma_{\text {Ğl }}^{2}$ et $\sigma_{\sigma_{2}}^{2}$ étant respectivement la variance génétique chez les mâles et chez les femelles

Nos données ne permettent qu'une évaluation approximative de $\sigma_{\text {G }}^{2} \mathrm{~s}^{\prime}:$ D'une part, $n$ n'est pas constant d'un groupe à l'autre et nous l'avons remplacé par l'effectif moyen sur l'ensemble des groupes; d'autre part, nous avons utilisé, pour $\sigma_{\mathrm{G} 1}$ et $\sigma_{\mathrm{G}_{2}}$, non des estimations exactes, mais des ordres de grandeur par excès, en multi- 
pliant les variances phénotypiques obtenues par une valeur d'héritabilité prise égale pour les deux sexes et estimée à 0,50 .

Dans le cas des poids à 4 semaines, le F moyen estimé sur l'ensemble des années est égal à $I, 73, n$ moyen est proche de 32 , le rapport $\sigma_{G}^{2} \mathrm{~s} / \sigma^{2}$ est trouvé égal à o,oI 4 et le facteur de correction a pour valeur $0,003 \sigma^{2}$, relativement faible devant celle de $\frac{\sigma_{\mathrm{G} S}^{2}}{\sigma^{2}}$

Pour le taux de croissance entre 4 et 8 semaines, $F=1,47$ dans l'ensemble. $\frac{\sigma_{\mathrm{G}} \mathrm{s}^{2}}{\sigma^{2}}=0,046$, le terme correctif ayant une valeur très faible de l'ordre de $0,00 I$.

Pour le poids à 8 semaines, $\mathrm{F}=\mathrm{I}, 43$ au total, $\frac{\sigma_{\mathrm{G}}^{2}}{\sigma^{2}}=0,0083$, le terme correctif étant de 1'ordre de 0,0035 .

Quant aux poids adultes, étant donnés les $\mathrm{F}$ assez importants, le terme correctif est faible comparé au rapport $\sigma_{\mathrm{G}}^{2} \mathrm{~s} / \sigma^{2}$.

En fin de compte, les termes correctifs, sans être totalement négligeables, ne paraissent pas suffire à annuler les variances "interaction » observées : celles-ci ont une existence réelle, tout en ne représentant qu'un pourcentage assez faible de la variance totale.

Les variations du dimorphisme sexuel pour le poids suivant la famille peuvent recevoir deux interprétations principales, d'ailleurs non exclusives l'une de l'autre : existence de gènes à manifestation différente suivant le sexe, ou à répartition différente dans les deux sexes. Dans nos données, relatives à une variation polygénique, on ne peut faire la part qui revient à l'une ou l'autre hypothèse.

La première possibilité peut correspondre aussi bien à des gènes autosomaux qu'à des gènes liés au sexe : on connaît depuis longtemps des exemples de gènes à effet dépendant du sexe ou limité à un sexe.

L'éventualité d'une répartition différente de certains gènes dans les deux sexes pourrait s'appliquer à des gènes liés au sexe, sous l'effet par exemple d'une sélection de direction ou d'intensité différente suivant le sexe pour le poids ou un caractère en corrélation avec lui, l'effet ne se maintenant pas, d'ailleurs, si une telle sélection cesse. Or, dans la pratique, la sélection exercée sur le poids est en général plus intense chez les coqs.

Le fait, suggéré par nos données et celles d'autres auteurs, d'une variabilité un peu plus grande par familles de mères que par pères s'expliquerait d'une façon simple par cette différence d'intensité de sélection entre sexes, comme l'ont suggéré SHAKLEE et al. (I952).

Deux autres causes non vérifiables actuellement, mais théoriquement possibles, de variations dans le dimorphisme sexuel ne peuvent, enfin, être écartées a priori : celle d'effets maternels sur la croissance, dont la différence suivant le sexe pourrait varier sous l'influence de facteurs génétiques ou non; celle de variations entre mères de 1'hétéro-chromosome $\mathrm{W}$ ayant une répercussion sur leur taille et celle de leurs filles à qui ce chromosome est transmis. L'existence du chromosome $W$ chez la poule semble en effet admise maintenant (OWEN, I965) et, d'autre part, on a suggéré dans d'autres espèces, et notamment chez 1'Homme, la possibilité d'effets quanti- 
tatifs associés à des variations de l'hétérochromosome $\mathrm{Y}$. De toutes façons, ces deux possibilités pourraient correspondre à la présence constatée de variations plus grandes entre mères qu'entre pères, et suggéreraient une sélection entre familles de frèressœurs.

Rę̧u pour publication en novembre 1971.

\section{REMERCIEMENTS}

Nous remercions le docteur L.-P. Cochez, Station expérimentale d'Aviculture du Magneraud, d'avoir mis à notre disposition des données utilisées dans ce travail.

\section{SUMMARY}

\section{DIFFERENCES BETWEEN FAMILIES FOR SEXUAL, DIMORPHISM OF WEIGHT AT A GIVEN AGE IN THE FOWI}

Differences of limited magnitude but highly significant were found between sires and dams within sires for sexual dimorphism of 4 week, 8 week and Io month body weight as well as for growth from 8 weeks to Io months. These differences are shown in strains of various origin, by testing family $\times$ sex interactions. The genetic interpretation (genes with sex-conditioned manifestation or sex-linked genes) is discussed. A scale effect due to the different variance for each sex is apparently unable to account entirely for them.

\section{RÉFÉRENCES BIBLIOGRAPHIQUES}

Beilharz R. G., I96o. Research into sex linked control of body weight in poultry and rabbits. Proc. Aust. Soc. Animal prod., 111, I39-146.

BuVANENDRAN V., I969. The heritability and genetic correlations of sexual dimorphism for ro week weight in poultry. Br. Poult. Sci, 10, 32I-325.

Clark T. B., I960. Crossbreeding in turkeys. II. Summary on heritability, growth, and body caracteristics. W. Va. Agr. Exp. Sta. Bull., 455 T.

Eisen E. J., Legates J. E., I966. Genotype-sex-interaction and the genetic correlation between the sexes for body weight in Mus Musculus. Genetics, 54, 6rr-623.

Glazener E. W., Blow W. L., Bastian C. H., Dearstyne R. S., i95r. Effect of inbreeding on broiler weights and feathering in the fowl. Poult. Sci., 30, 108-1r2.

Godfrey E. F., r953. The genetic control of growth and adult body weight in the domestic fowl. Poult. Sci., 32, 248-259.

Horton I. F., G. Mc Bride, I964. Genetic factors affecting sex dimorphism in body weight of meat chickens. Proc. Austral. Poultry Sci. Conv., 1964, 79-81.

Hutt F. B., I959. Sex-linked dwarfism in the fowl. J. Hered., 50, 209-22r.

JAAP R. G., I969. Phenotypic effects of sex-linked dwarfing genes in chickens. Poult. Sci., 48, 1826 (abstr.).

Kinney T. B. Jr, Shoffner R. N., 1965. Heritability estimates and genetic correlations among several traits in a meat type Poultry population. Poult. Sci., 44, I 0z0-1 032.

Maw A. J. G., 1935. The inheritance of skeletal dimensions in the domestic fowl. Sci. agric., 16, 85-II2.

MÉrat P., I962. Quelques relations entre caractères extérieurs à hérédité simple et productivité. Proc. XIIth world's Poultry Congress, Sydney, 1, 7I-76. 
MERRITT E. S., 1967. Genetic parameter estimates for growth and reproductive traits in a random bred control strain of meat type fowl. Poult. Sci., 46, r90-I99.

Moreng R. E., Thornton P. A., I958. Hybridization through inbreeding and strain crossing of turkeys. XIth World Poultry Congress, Mexico.

Owen J. J. T., I965. Karyotype studies on Gallus Domesticus. Chromosoma, 16, 6or-6o8.

Shaklee W. E., C. W., Knox S. J., Marsden I952. Inheritance of the sex difference of body weight in turkeys. Poult. Sci., 31, 822-825.

Snedecor G. W., Cochran W. G., 1967. Statistical methods, 6e Ed. Iowa State College press, Ames, Iowa.

Stonaker H. H., r963. A genetic hypothesis for sex-Mating system interaction in growth of cattle and poultry. J. animal Sci., 22, 320-325.

Wessel s J. P. H., Wilbraham B. O., r962. The production of table ducks by crossing egg-laying with table-type ducks. S. Afr. J. agric. Sci., 5, 67 I-672.

White J. M., Eisen E. J., Legates J. E., I968. Heterogametic Heterosis in body weight of mice. J. animal Sci., 27, I I 30 (abstr.).

Yoshida S., Collins W. M., r967. Individual Selection for 4 week body weight in japanese quail in relation to sex dimorphism. Poult. Sci., 46, I34 I (abstr.). 